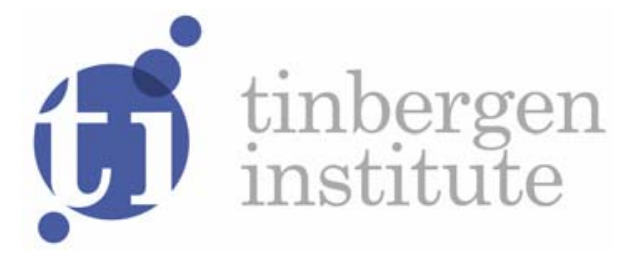

\title{
Social Network Effects and Green Consumerism
}

\author{
Dominic Hauck ${ }^{1}$ \\ Erik Ansink ${ }^{1,2}$ \\ Jetske Bouma3 \\ Daan van Soest ${ }^{4}$
}

' Faculty of Economics, VU University Amsterdam, the Netherlands;

2 Tinbergen Institute, the Netherlands;

3 PBL Netherlands Environmental Assessment Agency, the Netherlands;

4 Tilburg University, the Netherlands. 
Tinbergen Institute is the graduate school and research institute in economics of Erasmus University Rotterdam, the University of Amsterdam and VU University Amsterdam.

More TI discussion papers can be downloaded at http://www.tinbergen.nl

Tinbergen Institute has two locations:

Tinbergen Institute Amsterdam

Gustav Mahlerplein 117

1082 MS Amsterdam

The Netherlands

Tel.: +31(0)205251600

Tinbergen Institute Rotterdam

Burg. Oudlaan 50

3062 PA Rotterdam

The Netherlands

Tel.: +31(0)10 4088900

Fax: $+31(0) 104089031$

Duisenberg school of finance is a collaboration of the Dutch financial sector and universities, with the ambition to support innovative research and offer top quality academic education in core areas of finance.

DSF research papers can be downloaded at: http://www.dsf.nl/

Duisenberg school of finance

Gustav Mahlerplein 117

1082 MS Amsterdam

The Netherlands

Tel.: +31(0)20 5258579 


\title{
Social Network Effects And Green Consumerism*
}

\author{
Dominic Hauck ${ }^{\dagger}$ Erik Ansink Jetske Bouma $^{\S}$ Daan van Soest
}

December 2, 2014

\begin{abstract}
One of the drivers of green consumerism are social network externalities that are associated with buying 'green'- because green consumerism is fashionable, or because of reputation effects. We analyze how the strength of this social network effect impacts green consumerism, environmental externalities and total welfare. We discuss a model where products are differentiated according to their environmental quality, where the production of green products generates positive externalities to all, and where those consumers purchasing a green product variety receive the additional benefits of being a member of the network of green consumers. Depending on the strength of the social network effect, we show that (a) firms may produce lower quality, (b) the market may generate fewer positive environmental externalities, and (c) total welfare may deteriorate. The main policy implication is that if there is a network effect, regulators should choose a stricter minimum environmental quality standard.
\end{abstract}

Keywords: Quality Differentiation, Social Network Effect, Minimum Environmental Quality Standard.

JEL Codes: D11, L15, Q31.

\section{Corresponding author:}

Dominic Hauck

Institute for Environmental Studies (IVM), VU University Amsterdam

De Boelelaan 1087, 1081 HV Amsterdam, The Netherlands

E-mail: dominic.hauck@vu.nl

*We thank Harold Houba for helpful comments. Dominic Hauck, Erik Ansink and Jetske Bouma gratefully acknowledge financial support from the Netherlands Organisation for Scientific Research (NWO) as part of the Biodiversity Works Programme. Erik Ansink and Daan van Soest gratefully acknowledge receiving funding from the European Commission's Seventh Framework Program (FP7/20072013) under, respectively, grant agreement No. 269788 and grant agreement No. 613420.

${ }^{\dagger}$ Institute for Environmental Studies (IVM), VU University Amsterdam.

$\ddagger$ Department of Spatial Economics and IVM, VU University Amsterdam and Tinbergen Institute.

$\S$ Netherlands Environmental Assessment Agency (PBL) and IVM, VU University Amsterdam.

`Department of Economics and Tilburg Sustainability Center, Tilburg University. 


\section{Introduction}

Over the past few decades, the degree of environmental concern of consumers has been increasing and consumers are willing to pay more for products with a higher environmental quality (Flash Eurobarometer, 2009). A key driver of private consumers' decisions to purchase green product varieties is the "warm glow" associated with the consumption of less polluting goods (Andreoni, 1990). Researchers have also noted the existence of social network effects, such as the so-called bandwagon effect, defined by Leibenstein (1950) as "...the extent to which the demand for a commodity is increased due to the fact that others are also consuming the same commodity." The demand for green product varieties may be subject to a bandwagon effect because of signaling effects (including reputation building, see for example Nowak and Sigmund, 2005), or because green consumerism is just becoming more fashionable. While empirical research has indicated that such bandwagon effects increase willingness to pay for environmentally friendly products (Carlsson et al., 2010) as well as average donations in the context of charitable giving (Frey and Meier, 2004), their impact on the environmental quality of products offered on a market and on the environmental performance of such a 'green' market is not clear.

In this paper we analyze the consequences of a social network effect in green consumerism on (i) the greenness of the products produced, (ii) environmental externalities, and (iii) total welfare, consisting of producer surplus, consumer surplus, and the public benefits associated with environmental protection. We develop a model of a market in which three varieties of a specific product are sold: two green varieties (of differing levels of greenness), and a non-green product variety, the 'brown' one. The green firms act as duopolists competing in both prices and the greenness of the variety they produce, and the residual demand is satisfied by the brown firm. Consumers differ in the amount of warm glow they receive from consuming a product with a specific greenness, they equally benefit from impact of green consumerism on environmental externalities independent of whether they purchased a green or the brown variety, and those who purchased one of the two green varieties benefit from the social network effect associated with green 
consumerism.

Our results are as follows. Consistent with intuition, we show that the stronger the social network effect, the larger the market share of green product varieties in the market. However, and more surprisingly, we find that a stronger social network effect tends to reduce positive environmental externalities, and also that it may decrease total welfare. The policy implication is quite surprising too; we show that the stronger the social network effect, the more important it is for the regulator (or NGO) to set higher minimum standards. ${ }^{1}$

The main intuition for our results is the following. Green firms have an incentive to differentiate their qualities more if consumers benefit from a social network effect. Particularly, the firm producing the low-green product variety (the low-green firm) sets a lower environmental quality than in absence of the network effect because this allows it to (a) escape tight competition with the firm producing the high-green product variety (the high-green firm), (b) save on production costs, and (c) attract additional consumers who would otherwise purchase brown products. If, in addition to this, the quality choice of the high-green firm is not affected by the network effect (which we will see confirmed in this paper), average green product quality decreases. We show that the decline in average quality is not always compensated by the increased size of the green market due to brown consumers switching to green. Hence, the strategic choice of quality in the absence of the network effect may lead to environmentally inferior results as compared to models with environmental product differentiation but without network effects, as discussed by Cremer and Thisse (1999) among others. Our results are also complementary to other mechanisms that may reduce the positive impact of green consumerism on the environment, including the possibility that green consumerism may crowd out other voluntary attempts to clean up the environment (Kotchen, 2006).

Our paper differs from the extant literature in several respects. First, generalizing Brécard (2013), we endogenize market coverage, allowing for the possibility that the green market is not covered when consumers benefit from network effects. That is, a

\footnotetext{
${ }^{1}$ Our results concerning the minimum environmental quality standard hold equally well for ecolabels when considering standards and labels to be equivalent. Notably, both measures define a certain minimum quality level, which needs to be met for a product to be considered as environmentally friendly.
} 
fraction of consumers may choose to consume brown rather than (low or high) green. The importance of allowing for a non-covered green market is clear when considering realworld situations. Despite the increasing share of green products in consumer purchases, in most countries the bulk of consumers still purchases uncertified foods. Brécard (2013) indicates that the results obtained from a model of a non-covered green market may differ substantially from a covered green market model, which we see confirmed in the paper at hand. Particularly, Brécard (2013) shows that in a covered green market, the network effect does not affect the total level of environmental externalities which contrasts our findings.

Second, contrarily to Cremer and Thisse (1999), Brécard (2013) and Bansal and Gangopadhyay (2003), who discuss taxation and subsidization of quality production, we focus our discussion on optimal minimum environmental standards that aim to maximize total welfare. Evaluation of the welfare consequences of a minimum environmental quality standard in this paper confirms the findings of Ronnen (1991), who shows that welfare can be increased by choosing a stricter quality standard in the absence of network effects.

Third, based on a model of a non-covered market proposed by Choi and Shin (1992), Kuhn (2007) also assesses the effects of minimum quality standards. However, rather than focusing on social network effects, Kuhn (2007) discusses the situation where consumers derive a certain baseline profit from buying a product. Kuhn (2007) derives three types of equilibria and shows that the low quality firm may gain market dominance if this baseline benefit is large enough.

Finally, Friedman and Grilo (2005) analyze a model of horizontal product differentiation with social network effects where the number of firms is endogenous. Contrarily to Friedman and Grilo (2005), but in line with many papers on vertical product differentiation (e.g. Brécard, 2013, Kuhn, 2007, Conrad, 2005, Moraga-González and PadrónFumero, 2002, Cremer and Thisse, 1999), the paper at hand builds on the assumption of duopolistic competition on the market for green products.

The setup of our paper is as follows. In the next section, we present the formal version of a model with vertical product differentiation as discussed in the beginning of 
this introduction. In Section 3, we solve the game and derive one particular equilibrium. In Section 4, we further characterize this equilibrium to derive our main results. We analyze the effects of minimum environmental quality standards in Section 5, which requires further analysis of other equilibria. Section 6 concludes.

\section{A model with vertical product differentiation}

Consider an economy consisting of a unit mass of consumers and three types of firms. The consumers only differ in their marginal willingness to pay for green products, $\theta$. Of the three types of firms, one labeled $h$ produces a high-green variety of a good, one labeled $l$ produces a low-green variety, and a third type (labeled $b$ ) produces a brown variety. We use $q_{i}$ to denote the environmental quality (or, interchangeably, the greenness) of the product variety $i$, and $p_{i}$ to denote its sales price. The market share of the two green product varieties is denoted by $d_{i}(i=\{l, h\})$, and the market share of the brown variety is then equal to $1-d$, where $d \equiv d_{l}+d_{h}$. Finally, the overall quality of the environment in the economy is defined as the weighted sum of positive environmental externalities due to production.

$$
E=d_{l} q_{l}+d_{h} q_{h}+(1-d) q_{b}
$$

Normalizing the greenness of the brown variety to zero, Equation (1) implies that we evaluate the environmental consequences of green consumption against a benchmark of $E=0$ : zero environmental externalities if all consumers consume brown. ${ }^{2}$

Regarding the demand side of the economy, consumers derive utility $(U)$ from the greenness of the good they consume $\left(q_{i}\right)$, from environmental externalities $(E)$, and from the network benefits associated with the total amount of green product varieties consumed $(d)$, which consumers only receive when they themselves purchase a green variety; they derive dis-utility from the amount of money they need to spend on purchasing the good they consume $\left(p_{i}\right)$. For tractability, the utility function is assumed to be separable

\footnotetext{
${ }^{2}$ Because of this benchmark choice, green consumption generates positive externalities to all consumers. Obviously, we could also have modeled the environmental impact of green consumption as resulting in fewer negative externalities compared to brown consumption.
} 
in each of these components:

$$
U=\theta q_{i}+\delta E+\lambda \alpha_{i} d-p_{i}
$$

where $i \in\{b, l, h\}$ indicates the consumed product variety and $\alpha_{i}$ is a dummy variable indicating membership of the network of green consumers. Only those consumers who purchase a green product variety benefit from the green consumers' social network effect (that is, $\alpha_{i}=1$ if $i=\{l, h\}$, and zero otherwise), and they equally do so independent of whether they purchase the low- or high-green variety. ${ }^{3}$ As stated above, parameter $\theta$ represents the marginal willingness to pay (MWTP) of the individual consumer for the greenness of the good she is consuming; $\theta$ is assumed to be uniformly distributed over the mass of consumers, normalized on support $[0,1]$. Next, parameter $\delta$ denotes the marginal benefit obtained from environmental externalities $E$, and $\lambda$ represents the marginal benefits the consumer derives from (being part of) the network of green consumers, $\alpha_{i} d .^{4}$

On the supply side, we assume that there are two competing firms on the green market, while the brown market is characterized by perfect competition. ${ }^{5}$ In our model, the green duopolists first choose the greenness (or environmental quality) of their products, and subsequently compete in prices. By definition, the level of greenness produced by one firm is (weakly) higher than that produced by the other, and we refer to the former (latter) as the high- (low-)green firm. We also assume there is a maximum to the greenness of the produced varieties, $\bar{q}$, and sometimes also a minimum level, $\underline{q}$, which may be imposed by the government or a certifying agency. Without loss of generality, we thus have $\underline{q} \leq q_{l} \leq q_{h} \leq \bar{q}$, with at least one of the inequalities being strict. Next, in line with Kuhn (2007) we assume that the variable costs of producing a product variety depend on its quality, i.e. the cost of producing a variety with quality $q_{i}$ is given by $c q_{i}$,

\footnotetext{
${ }^{3}$ It is certainly possible that the network effects are product specific. Allowing this differentiation obstructs analytical tractability of the model, and is left for future research.

${ }^{4}$ We acknowledge that the exact size of the network may be hard to observe for individual consumers. As a result, perceptions or beliefs of demand may play a crucial role here. These beliefs might be driven by various factors such as the greenness of the product varieties. In this paper we ignore such complicating factors.

${ }^{5}$ We assume that none of the brown firms will invade the green market.
} 
where $c \in[0,1)$. Firms are assumed to set prices and qualities to maximize their profits:

$$
\pi_{i}=d_{i}\left(p_{i}-c q_{i}\right)
$$

Because $q_{b}$ is normalized to zero and because the brown market is characterized by perfect competition, we have $p_{b}=0$ in equilibrium.

\section{Analysis}

We use the equilibrium concept of sub-game perfect Nash equilibrium (SPNE) (Selten, 1975) to derive the greenness of the varieties, their prices and quantities demanded. Recall that the two firms producing the green varieties first choose the greenness of their products (Stage I) and subsequently compete in prices (Stage II), which determines demand for the two green product varieties. We first derive the demand functions (taking prices and qualities of the product varieties as given) in Section 3.1. Subsequently, using backward induction, we solve Stage II in Section 3.2, and Stage I in Section 3.3.

\subsection{Deriving the demand functions for each of the two green varieties}

Consumers only differ in their marginal willingness to pay for the environmental quality of the product they buy, $\theta$. Consumers with a $\theta$ close to 1 are more likely to buy the high-green variety, while consumers with a $\theta$ close to zero are more likely to purchase the brown variety; see Equation (2). Market shares for each of the three product varieties can be derived by identifying (i) the consumer who is indifferent between purchasing the brown and low-green variety, and (ii) the consumer who is indifferent between buying the low-green and high-green variety. Referring to these two consumers as $\hat{\theta}_{l}$ and $\hat{\theta}_{h}$ respectively, the shares of brown, low-green and high-green products in the market are $1-d=\hat{\theta}_{l}, d_{l}=\hat{\theta}_{h}-\hat{\theta}_{l}$ and $d_{h}=1-\hat{\theta}_{h} ;$ see also Figure 1 .

Let us first derive $\hat{\theta}_{l}$. All consumers benefit from the environmental externalities independent of whether they purchase a green or brown product variety, and the social network effect is equally large for all consumers purchasing green products, whether it is the low- or the high-green variety. Using (2), a consumer's utility when purchasing 
the brown variety is equal to just $\delta E$ (because $p_{b}=0$, and $\alpha_{b}=0$ ), while her utility when consuming the low-green variety is equal to $\hat{\theta}_{l} q_{l}+\lambda d+\delta E-p_{l}$, where $d=1-\hat{\theta}_{l}$. Equating the two, we have

$$
\hat{\theta}_{l}=\frac{p_{l}-\lambda}{q_{l}-\lambda} .
$$

Note that, all else equal, we should have that a very small increase in this consumer's MWTP for greenness should unambiguously increase her utility. The reason is that if consumer $\hat{\theta}_{l}$ would have slightly stronger preferences for greenness, the size of the network does not decrease (as she keeps on buying $l$ ), while she attaches a higher value to the greenness of the variety she purchases. Substituting $\alpha=1, \theta=\hat{\theta}_{l}$ and $d=1-\hat{\theta}_{l}$ into (2) and taking the first derivative, we find that $d U\left(\hat{\theta}_{l}\right) / d \theta>0$ if and only if $q_{l}>\lambda$. Given that $q_{l}$ is an endogenous variable and taking into account the possible constraints on the greenness levels (and then especially the condition that $q_{l} \geq \underline{q}$ ), we need to check whether all equilibria satisfy Condition 1:

Condition 1. $\lambda<\max \left[q_{l}, \underline{q}\right] \leq q_{h}$

Given Condition 1, Equation (4) then indicates that the green market is covered (not covered) if $p_{l}=\lambda\left(p_{l}>\lambda\right)$ : all consumers with $0 \leq \theta \leq \hat{\theta}_{l}$ (weakly) prefer consuming the brown variety.

Having derived $\hat{\theta}_{l}$, we now turn to determining $\hat{\theta}_{h}$, the consumer who is indifferent between consuming the low-green and the high-green variety. Here, the environmental benefit is independent of consumed product variety (because it is a pure externality), and also the network benefits are the same independent of the consumed green product variety. Using (2), the difference in utilities from buying the low-green or the highgreen product only depends on the (valuation of the) difference in qualities and on the difference in purchase prices (see $(2)$ ), and hence $\hat{\theta}_{h}$ is equal to

$$
\hat{\theta}_{h}=\frac{p_{h}-p_{l}}{q_{h}-q_{l}}
$$

From Equations (4) and (5) we have that all consumers with $1 \geq \theta>\hat{\theta}_{h}$ are strictly better off consuming the high-green variety, while all consumers with $\hat{\theta}_{l} \leq \theta \leq \hat{\theta}_{h}$ end up purchasing the low-green variety. 


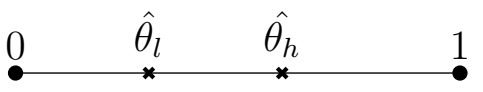

Figure 1: Graphical representation of the market shares of the brown, low-green and high-green product varieties, where all consumers with $\theta<\hat{\theta}_{l}$ purchase the brown variety, all those with $\theta>\hat{\theta}_{h}$ purchase the high-green variety, and the remaining consumers purchasing the low-green variety.

Remark 1. Demand for each product variety does not depend on the externalities generated by consumption of green products. Not individual, but aggregate demand determines the market equilibrium (Cremer and Thisse, 1999). This model feature is in line with many green markets, on which individual consumption decisions do not affect qualities and prices offered.

Next, using the derived demand functions for the two green product varieties, $d_{l}=$ $\hat{\theta}_{h}-\hat{\theta}_{l}$ and $d_{h}=1-\hat{\theta}_{h}$, we solve the model subject to Condition 1 .

\subsection{Equilibrium prices and demands - Stage II}

Given equilibrium qualities from Stage I, firms compete in prices in Stage II. Taking the first derivative of the firms' profit functions (3) with respect to price and using asterisks to denote profit-maximizing values, we have: ${ }^{6}$

$$
\begin{aligned}
& p_{l}^{*}=\frac{1}{a}\left(\lambda\left[q_{h}(1-c)-q_{l}(2 c+1)\right]+q_{l}\left[q_{h}(3 c+1)-q_{l}\right]\right), \\
& p_{h}^{*}=\frac{1}{a}\left(q_{l}\left[\left(q_{h}(c+2)\right)-\lambda(c-1)\right]-2 q_{h}\left(\lambda+q_{h}\right)(c+1)\right),
\end{aligned}
$$

where $a \equiv 4 q_{h}-q_{l}-3 \lambda>0$, due to the inequalities in Condition 1 .

Substituting (4) and (5) into $d_{l}=\hat{\theta}_{h}-\hat{\theta}_{l}$ and $d_{h}=1-\hat{\theta}_{h}$ and using (6) and (7), we find the following equilibrium demand functions for the two green product varieties:

$$
\begin{aligned}
d_{l}^{*} & =\left(\frac{1}{a}\right)\left(\frac{1}{q_{l}-\lambda}\right)(1-c)\left(q_{h}-\lambda\right)\left(q_{l}+\lambda\right), \\
d_{h}^{*} & =\left(\frac{1}{a}\right)(1-c)\left(2 q_{h}-\lambda\right) .
\end{aligned}
$$

Given $a$ and given the inequalities in Condition 1, we find that all terms in both $d_{l}^{*}$ and $d_{h}^{*}$ are non-negative so that qualities demanded are positive. Following standard intuition,

\footnotetext{
${ }^{6}$ Most derivations in the paper involve relatively uncomplicated substitution and optimization. They are available upon request.
} 
demand for both green product varieties decreases in marginal production cost $c$. In the limit where $c$ approaches 1 , there is no demand for either of the green product varieties, because costs - and therefore prices - become prohibitively high. Finally, note that $d_{h}^{*}$ is increasing in $q_{l}$ (via the term $a$ ); a relation that we will use later in the paper.

\subsection{Equilibrium quality and profits - Stage I}

We now turn to the firms' choice of qualities in Stage I. Substituting (6)-(9) into (3) we have

$$
\begin{aligned}
\pi_{l} & =\frac{(1-c)^{2}\left(q_{h}-q_{l}\right)\left(q_{h}-\lambda\right)\left(\lambda+q_{l}\right)^{2}}{\left(q_{l}-\lambda\right)\left(3 \lambda+q_{l}-4 q_{h}\right)^{2}}, \\
\pi_{h} & =\frac{(1-c)^{2}\left(q_{h}-q_{l}\right)\left(\lambda-2 q_{h}\right)^{2}}{\left(3 \lambda+q_{l}-4 q_{h}\right)^{2}} .
\end{aligned}
$$

Both profits are strictly positive if Condition 1 holds. Moreover, $\pi_{l}$ and $\pi_{h}$ are decreasing in $c$.

To determine equilibrium qualities (or greenness), we maximize the firms' profit functions (10) and (11) with respect to quality. We obtain the following results.

Lemma 1. For $\lambda \in\left[0, \frac{2}{25} \bar{q}\right]$, equilibrium qualities are given by

$$
\left(q_{l}^{\star}, q_{h}^{\star}\right)=\left(q_{l}^{\prime}\left(q_{h}, \lambda\right), \bar{q}\right)
$$

where

$$
q_{l}^{\prime}\left(q_{h}, \lambda\right)=\frac{\left(q_{h}-\lambda\right) \sqrt{25 \lambda^{2}-52 \lambda q_{h}+4 q_{h}^{2}}+2\left(3 \lambda q_{h}+q_{h}^{2}-2 \lambda^{2}\right)}{7 q_{h}-3 \lambda} .
$$

In this equilibrium, the green market is not covered and Condition 1 is satisfied.

Proof. We prove this result by showing that for all $\lambda \in\left[0, \frac{2}{25} \bar{q}\right]$, we have that a) $q_{l}^{\star}$ and $q_{h}^{\star}$ are indeed optimal responses to each other, b) the green market is not covered, i.e. $\hat{\theta}_{l}>0$, and c) Condition 1 holds.

Concerning part a) we maximize Equation (10) with respect to $q_{l}$ and solve. This results in a hyperbolically shaped reaction correspondence, i.e. there are two solutions 
of $q_{l}$ for all $\lambda \in\left[0, \frac{2}{25} q_{h}\right]$. In Appendix A we show that solutions on the upper branch of the hyperbola dominate those on the lower branch. For $\lambda>\frac{2}{25} q_{h}$, the best-response function is not real-valued. ${ }^{7}$

If we optimize Equation (11) with respect to $q_{h}$, we do not obtain a real valued best-response function for $\lambda<\frac{23}{25} q_{l}{ }^{8}$ Hence, we continue the analysis by directly evaluating the first order condition of profit maximization by the high-green firm under the condition that the low-green firm maximizes profit. Particularly, we substitute $q_{l}^{\prime}\left(q_{h}, \lambda\right)$ into the derivative of the profit function of the high-green firm, (11), which yields

$$
\begin{aligned}
\frac{\partial \pi_{h}\left(q_{h}\right)}{\partial q_{h}} & =(1-c)^{2} \cdot\left(\lambda-2 q_{h}\right) \cdot\left(3 \lambda-7 q_{h}\right) \\
& \cdot\left(a\left(47 \lambda^{2}-101 q_{h} \lambda+26 q_{h}^{2}\right)+\lambda\left(251 \lambda^{2}-903 q_{h} \lambda+972 q_{h}^{2}\right)-340 q_{h}^{3}\right) \\
& \cdot\left(\lambda-q_{h}\right)^{-2} \cdot\left(a+13 \lambda-26 q_{h}\right)^{-3},
\end{aligned}
$$

where $a \equiv \sqrt{25 \lambda^{2}-52 q_{h} \lambda+4 q_{h}^{2}} \in\left[0,2 q_{h}\right]$ for all $\lambda \in\left[0, \frac{2}{25} \bar{q}\right]$.

Except $(1-c)^{2}, a,\left(\lambda-q_{h}\right)^{2}$, the terms in (13) are negative in the interval $\lambda \in\left[0, \frac{2}{25} \bar{q}\right]$. This implies that $\frac{\partial \pi_{h}\left(q_{h}\right)}{\partial q_{h}}>0 .{ }^{9}$ Hence, given the optimal choice of the low-green firm, the high-green firm always wants to maximally differentiate by producing the technically maximum feasible quality $\bar{q}$.

Next, we turn to part b) of the proof to show that $\hat{\theta}_{l}>0$ for all $\lambda \in\left[0, \frac{2}{25} \bar{q}\right]$. From the proof of Proposition 1 in Section 4 below, we learn that $\frac{\partial d}{\partial \lambda}>0$, i.e. market coverage is increasing in $\lambda$. As a result, if the green market is not covered for larger values of $\lambda$, it will also not be covered for small values of $\lambda$. Therefore, we only need to check if the green market is covered for the largest real-valued solution of $\lambda$ where $\lambda=\frac{2}{25} \bar{q}$. We do so by substituting this value of $\lambda$, together with equilibrium qualities $q_{h}^{\star}=\bar{q}$ and

\footnotetext{
${ }^{7}$ We can show that the first order condition of the low-green firm is decreasing in $q_{l}$ for a broad range of parameters if $\lambda>\frac{2}{25} q_{h}$, i.e. the low-green firm wants to maximally differentiate her product from the high-green firm. This gives rise to additional equilibria. See also Footnotes 8 and 11.

${ }^{8}$ The reaction function of the high-green firm for $\lambda \geq \frac{23}{25} q_{l}$ is

$$
q_{h}^{\prime}\left(q_{l}, \lambda\right)=\frac{1}{8}\left(7 \lambda+3 q_{l}-\sqrt{25 \lambda^{2}+2 q_{l} \lambda-23 q_{l}^{2}}\right) .
$$

${ }^{9}$ Note that the term $a\left(47 \lambda^{2}-101 q_{h} \lambda+26 q_{h}^{2}\right)+\lambda\left(251 \lambda^{2}-903 q_{h} \lambda+972 q_{h}^{2}\right)-340 q_{h}^{3}$ is strictly negative for all $\lambda \in\left[0, \frac{2}{25} \bar{q}\right]$.
} 


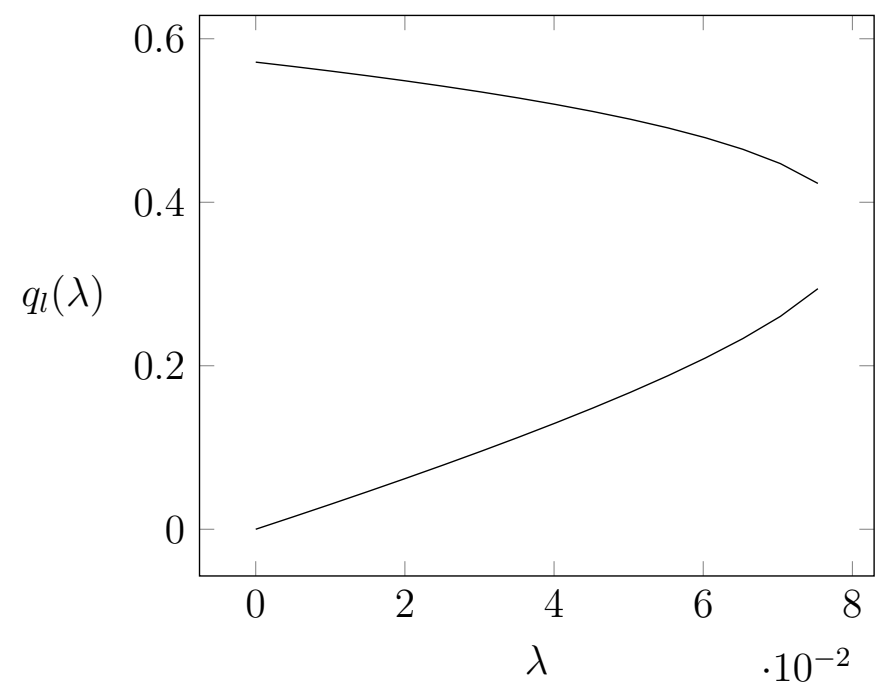

Figure 2: The low-green firm's reaction correspondence for $c=0$ and $q_{h}=\bar{q}=1$.

$q_{l}^{\star}=\frac{118}{325} \bar{q}$, and equilibrium price $p_{l}^{\star}$ from (6), into the indifference function (4). After substantial simplification, we obtain

$$
\hat{\theta}_{l}=\frac{91 c+1}{92} \in(0,1) \forall c \in[0,1)
$$

Because $\hat{\theta}_{l}$ is strictly positive, there is a strictly positive share of consumers that consume brown. The green market is not covered for any $c \in[0,1) \cdot{ }^{10}$

Finally, note that part a) and b) imply that $q_{l}^{\star}=q_{l}^{\prime}\left(q_{h}, \lambda\right)>\lambda \in\left[0, \frac{2}{25} \bar{q}\right]$, i.e. Condition 1 holds.

Many results discussed in the next section, Section 4 , are based on the fact that $q_{l}^{\star}$ is decreasing in $\lambda$. This relation is shown in Figure 2, where we plot the reaction correspondence for $q_{h}=\bar{q}=1$ (note that we can obtain similar hyperbolas for $\bar{q} \neq 1$ ). In Figure 2, the upper branch of the hyperbola represents the best reply by the low-green firm.

In the real world, the network effect of green consumerism is important, but as a driver of the preference for green goods it is likely to be relatively weak compared to the intrinsic preference for green consumption. Therefore, in the remainder of this paper

\footnotetext{
${ }^{10}$ It is not surprising that, according to Equation (14), $\hat{\theta}_{l}$ is increasing in $c$. As $c$ increases, prices of green products increase which implies that the consumer who is indifferent between buying low-green and brown shifts to the left in Figure 1, i.e. less people consume green for any level of $\lambda$.
} 
we discuss the impact of the network effect in the context of the equilibrium discussed in Lemma 1. Particularly, we focus on the region where $\lambda \in\left[0, \frac{2}{25} \bar{q}\right]$ and $\bar{q}$ is not too large. ${ }^{11}$ For example, if $\bar{q}=1$, the network effect is less than $16 \%$ of the average intrinsic preference for quality, which equals $\frac{1}{2}$.

\section{Results}

Regarding the impact of the strength of the network effect, $\lambda$, on environmental externalities and on total welfare, two effects are important. First, and not surprisingly, a higher level of $\lambda$ can be expected to increase the size of the market for green product varieties. In Proposition 1 we show that this is indeed the case. Second, the greenness of the two varieties are also a function of the strength of the social network effect, and we analyze this relation in Proposition 2. Subsequently, in Proposition 3 and Corollary 1, we show how the interplay between the demand expansion for green varieties and the change in the greenness of the varieties affects environmental externalities and total welfare.

Our first result is the following.

Proposition 1. The size of the total market share covered by green product varieties is increasing in the strength of the network effect, i.e. $\frac{\partial d}{\partial \lambda}>0, \forall \lambda \in\left[0, \frac{2}{25} \bar{q}\right]$ and given $\bar{q}=1$.

Proof. We have $d \equiv 1-\hat{\theta}_{l}$. Using (4), we have $\frac{\partial d}{\partial \lambda}=\frac{\partial\left(1-\hat{\theta}_{l}\right)}{\partial \lambda}=\frac{q_{l}^{\star}-p_{l}^{\star}}{\left(\lambda-q_{l}^{\star}\right)^{2}}$ which is positive if $q_{l}^{\star}>p_{l}^{\star}$.

Using equilibrium quantities and $\bar{q}=1$, we obtain $q_{l}^{\star}-p_{l}^{\star}=\frac{(1-c)\left(a(2 \lambda-3)+8 \lambda^{2}-13 \lambda-6\right)}{a+13 \lambda-26}$, which is strictly positive for all $c \in(0,1), \lambda \in\left[0, \frac{2}{25} \bar{q}\right]$ and where we have defined $a \equiv \sqrt{25 \lambda^{2}-52 \lambda+4}$.

The proof is based on the inequality $q_{l}^{\star}-p_{l}^{\star}>0$ for $\lambda \in\left[0, \frac{2}{25} \bar{q}\right]$. Simulations indicate that this inequality also holds for $\bar{q} \neq 1$, which generalizes the result of Proposition 1.

\footnotetext{
${ }^{11}$ Note that the results reported in Footnotes 7 and 8 give rise to additional equilibria, for larger values of $\lambda$, whose existence depends crucially on assumptions on $q$. One of these equilibria, an equilibrium of maximum product differentiation, is discussed in Section $\overline{5}$. In the other equilibrium, the high-green firm reduces her quality. These equilibria are qualitatively similar to those discussed by Kuhn (2007) and we refer the interested reader to this study.
} 
We will refer to the expansion of green market coverage due to the network effect, as the demand effect. To illustratively quantify the demand effect, we compare the model outcomes with and without a social network effect for $c=0, \bar{q}=1$. If $\lambda=0$, there is no network effect ${ }^{12}$, and we have $\left(d_{l}^{*}, d_{h}^{*}\right)=(0.29,0.58)$; if $\lambda=\frac{2}{25} \bar{q}$, we have $\left(d_{l}^{*}, d_{h}^{*}\right)=(0.42,0.57) \cdot{ }^{13}$

We proceed by evaluating the impact of the social network effect on the equilibrium average quality of the green product varieties, $q_{a} \equiv \frac{1}{2}\left(q_{h}+q_{l}\right)$, on the total amount of positive environmental externalities produced, and on total welfare.

Proposition 2. Average quality, $q_{a}$, is decreasing in the extent to which consumers benefit from the social network effect, $\lambda$, i.e.

$$
\frac{\partial q_{a}}{\partial \lambda}=\frac{1}{2} \frac{\partial q_{l}^{\star}}{\partial \lambda}<0 \quad \forall \lambda \in\left[0, \frac{2}{25} \bar{q}\right] .
$$

Proof. We plot the reaction correspondence for $\bar{q}=1$ in Figure 2. The upper branch of the hyperbola represents the best response function of the low-green firm. Simulations produce similar hyperbolas for various $\bar{q} \neq 1$. Note that the best response function does not depend on $c$.

Because $q_{l}^{*}(\lambda, \bar{q})$ is decreasing in $\lambda$ for all $\lambda \in\left[0, \frac{2 q}{25} \bar{q}\right]$ and because $q_{h}^{*}=\bar{q}$ is independent of $\lambda$, the equilibrium average quality, $q_{a}^{*}=\frac{1}{2}\left(q_{l}^{*}+q_{h}^{*}\right)$, is also decreasing in $\lambda$.

We will refer to the decrease of average quality due to the network affect, as the quality effect. To illustratively quantify the quality effect, we evaluate the two endpoints of the permitted interval for $\lambda,\left[0, \frac{2}{25} \bar{q}\right]$. We find that $q_{l}^{\star}(\bar{q}, \lambda=0)=\frac{4}{7} \bar{q}$, which confirms the findings of Choi and Shin (1992). At the maximum of the interval of permitted values for $\lambda, q_{l}^{\star}\left(\bar{q}, \lambda=\frac{2}{25} \bar{q}\right)=\frac{118}{325} \bar{q}$. Note that $\frac{4}{7}>\frac{118}{325}$, i.e. the low-green firm produces higher quality if $\lambda=0$ compared to $\lambda=\frac{2}{25} \bar{q}$.

Having identified the demand and quality effects in Propositions 1 and 2, we now proceed evaluating the impact of $\lambda$ on environmental externalities, and also on total welfare.

\footnotetext{
${ }^{12}$ This situation resembles the situation discussed by Choi and Shin (1992).

${ }^{13}$ Obviously, the difference in market shares, $d_{h}^{*}-d_{l}^{*}>0$, is maximal for $c=0 . d_{h}^{*}-d_{l}^{*}>0$ converges towards zero as $c$ approaches 1 because both market shares converge to zero.
} 


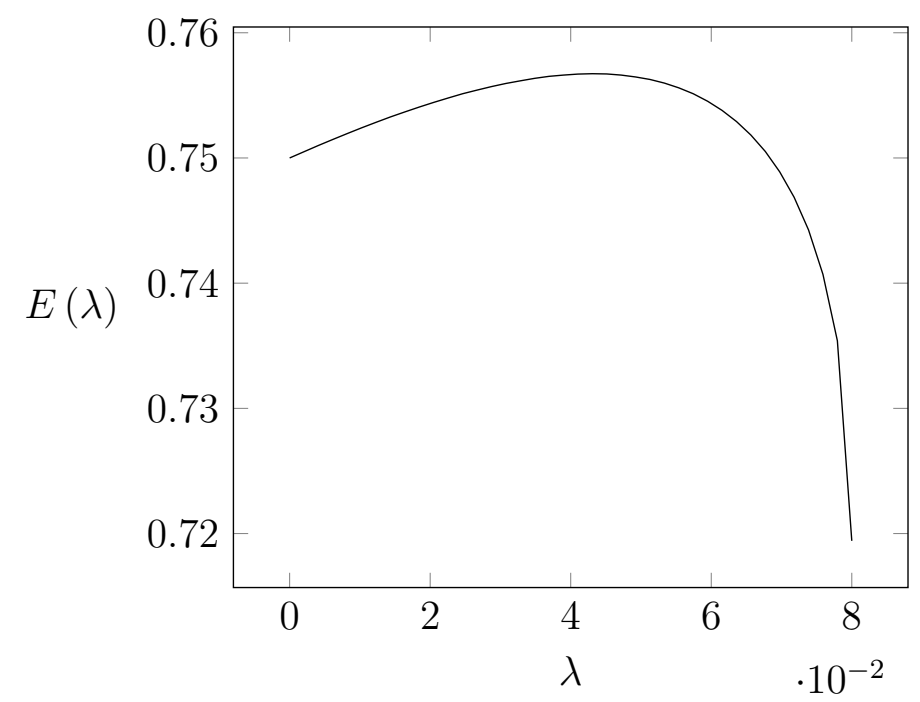

Figure 3: $E(\lambda)$ as a function of $\lambda \in\left[0, \frac{2}{25} \bar{q}\right]$ where $\bar{q}=1$ and $c=0$.

Regarding the relationship between $\lambda$ and $E$ — as defined in (1) — it is not immediately clear whether a higher $\lambda$ translates into a higher $E$. On the one hand, the increase in total market coverage due to the demand effect impacts $E$ positively (as discussed in Proposition 1), while the quality effect has the opposite impact (as discussed in Proposition 2). Figure 3 indicates that the impact of $\lambda$ on $E$ is ambiguous. In the following proposition, we further specify this.

Proposition 3. We state:

a) There exists some $\lambda \equiv \lambda_{E 0}$ such that $\frac{\partial E}{\partial \lambda}>0$ for $\lambda \in\left[0, \lambda_{E 0}\right)$ and $\frac{\partial E}{\partial \lambda}<0$ for $\lambda \in\left(\lambda_{E 0}, \frac{2}{25} \bar{q}\right]$

b) $E(\lambda=0)>E\left(\lambda=\frac{2}{25} \bar{q}\right) \forall c \in[0,1)$.

In words, there is some $\lambda_{E 0}$ such that $E$ is increasing for $\lambda<\lambda_{E 0}$ but decreasing for $\lambda>\lambda_{E 0}$. Hence, for $\lambda>\lambda_{E 0}$, the quality effect dominates the demand effect. Moreover, the total amount of positive environmental externalities generated in absence of the social network effect is higher than when the network effect is maximal.

Proof. In order to proof part a) we need to show that the $E$ function has a maximum at $\lambda_{E 0}$, i.e. that $\frac{\partial E}{\partial \lambda}$ is positive for small values of $\lambda$ but negative for large values of $\lambda$. According to the Intermediate Value Theorem, a polynomial that has different signs at 
two distinct values of its domain must have a zero. Now, it can easily be verified that the Intermediate Value Theorem holds by substituting equilibrium quantities into $\frac{\partial E}{\partial \lambda}$. Particularly, at $\lambda=0, \frac{\partial E}{\partial \lambda}>0$, and at $\lambda=\frac{2}{25} \bar{q}, \frac{\partial E}{\partial \lambda}=-\infty<0$ such that both conditions for the existence of the maximum are satisfied.

We can prove the second part of Proposition 3 by substituting equilibrium quantities into (1). We have $E(\lambda=0)=\frac{3}{4} \bar{q}(1-c)$. If $\lambda=\frac{2}{25} \bar{q}$, then substituting uSPNE equilibrium qualities $q_{l}^{\star}=\frac{118}{325} \bar{q}$ and $q_{h}^{\star}=\bar{q}$ as well as market shares (8) and (9) into (1) yields $E\left(\lambda=\frac{2}{25} \bar{q}\right)=\frac{827}{1150} \bar{q}(1-c)$. Comparing both outcomes we obtain $E(\lambda=0)=$ $\frac{3}{4} \bar{q}(1-c)>\frac{827}{1150} \bar{q}(1-c)=E\left(\lambda=\frac{2}{25} \bar{q}\right)$, which completes the proof.

For $\bar{q}=1$, Figure 3 shows that the maximum of the $E$ function is unique. We know that $c$ enters the polynomial linearly and the sign of $\frac{\partial E}{\partial \lambda}$ at $c=0$ and at $c=1$ coincide for any $\lambda$ in the relevant interval. Hence, $c$ does not affect the location of the zero of $\frac{\partial E}{\partial \lambda}$. Using a numerical approach, we obtain the zero of the derivative, $\lambda_{E 0} \approx 0.043 \bar{q} .{ }^{14,15}$

We find that the impact of $\lambda$ on $E$ is ambiguous, and hence the impact of $\lambda$ on total welfare is likely to be ambiguous too, as discussed in the following corollary:

Corollary 1. There exists some $\lambda \equiv \lambda_{W 0}$ such that $\frac{\partial W}{\partial \lambda}>0$ for $\lambda \in\left[0, \lambda_{W 0}\right)$ and $\frac{\partial W}{\partial \lambda}<0$ for $\lambda \in\left(\lambda_{W 0}, \frac{2}{25} \bar{q}\right], \bar{q}=1$.

In other words, there is some $\lambda_{W 0}(c, \delta)$ such that total welfare is decreasing in the social network effect for $\lambda>\lambda_{W 0}(c, \delta)$. The proof of this statement is based on the Intermediate Value Theorem and resembles the proof of Proposition $3{ }^{16}$ We obtain the zero of the derivative of the welfare function, $\lambda_{W 0}(c, \delta) \approx 0.0788$ in the relevant interval with a numerical approach.

In Figure 4, we display simulations of $\lambda_{W 0}(c, \delta)$ for $\bar{q}=1$ and various values of $c$ and $\delta$. The figure shows that if the costs of producing quality, $c$, are relatively high, total

\footnotetext{
${ }^{14}$ Obviously, $E$ is decreasing in $c$. As the production of environmental quality becomes more expensive, fewer environmental externalities will be generated.

${ }^{15}$ Establishing that the zero of $E$ is unique for $\bar{q} \in \mathbb{N}$ is complicated because the nominator of $\frac{\partial E}{\partial \lambda}$ is a high order polynomial, potentially having many zeros that may depend on $c$. However, simulations indicate that the zero of $E$ is unique for arbitrary $\bar{q}$.

${ }^{16}$ We obtain similar results for $\bar{q} \neq 1$ by simulation.
} 


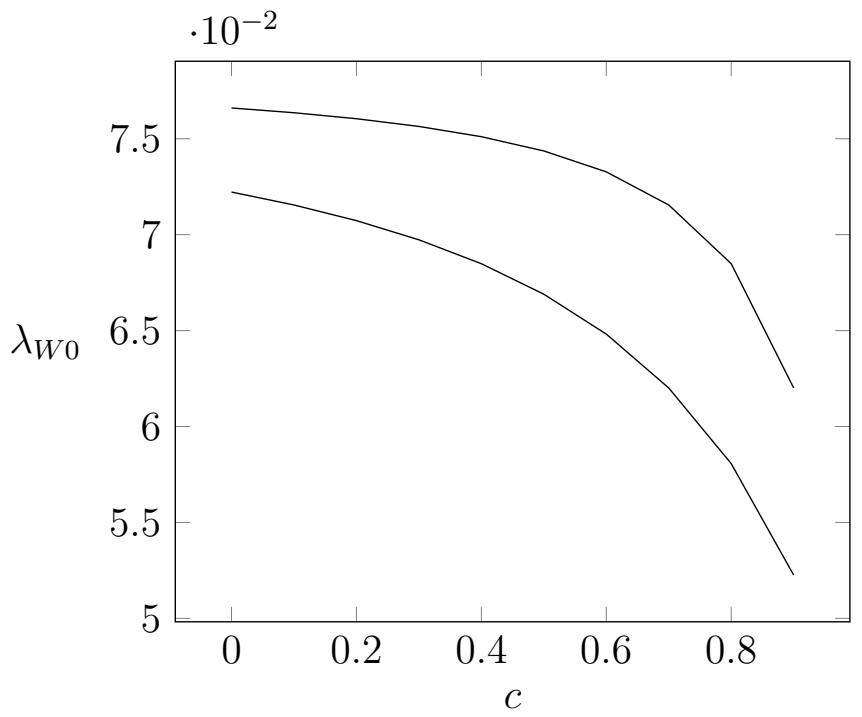

Figure 4: Zeros of $\frac{\partial W}{\partial \lambda}, \lambda_{W 0}$, as a function of $c$, with $\delta=.2$ (upper) and with $\delta=.6$, given $\bar{q}=1$

welfare decreases for lower values of $\lambda>\lambda_{W 0}$ for any $\delta$. The same is true for higher values of $\delta$ given $c$. Subsequently, we will discuss this result in more detail.

The loss of welfare for $\lambda>\lambda_{W 0}$, is due to a loss in consumer surplus, which can be written as,

$$
C S=\int_{\hat{\theta}_{l}}^{\hat{\theta}_{h}}\left[\theta q_{l}-p_{l}\right] d \theta+\int_{\hat{\theta}_{h}}^{1}\left[\theta q_{h}-p_{h}\right] d \theta+\lambda\left(1-\hat{\theta}_{l}\left(2-\hat{\theta}_{l}\right)\right)+\delta E,
$$

where the third term on the right hand side results from integration of the network effect over the total mass of green consumers. Again, with higher cost of producing quality, and with higher $\delta$, consumer surplus is negatively affected for smaller values of $\lambda$. Particularly, given $\bar{q}=1$ for $c=0, \delta=0$, consumer surplus is decreasing for $\lambda \gtrsim 0.0788$, while for $c=.9, \delta=1$, consumer surplus is decreasing for $\lambda \gtrsim 0.0441$. With higher $c$, aggregate demand for green goods must be smaller due to higher prices. This curbs the direct benefit consumers derive from the network effect, as captured in the third term on the right hand side of Equation (15). Simultaneously, the decrease in consumer surplus due to the quality effect remains unaffected by an increase in $c$. The same logic applies for higher values of $\delta$.

The decrease in consumer surplus is partially compensated by an increase in aggre- 
gate firm profits. Firms exploit stronger network effects by increased product differentiation. Particularly, the low-green firm increases differentiation by producing lower quality. Due to the demand effect, the low-green firm attracts more customers despite producing lower quality. More product differentiation results in relaxed price competition, and in Section 3.1, we showed that prices are indeed increasing in $\lambda$. Comparing prices for $\lambda=0$ (no network effect) and $\lambda=2 / 25$ (maximal network effect in the equilibrium with $\bar{q}=1)$, we find that $p_{l}^{*}(\lambda=0)<p_{l}^{*}\left(\lambda=\frac{2}{25}\right)$ for $c<0.053$. Hence, the low-green firm charges a higher price despite offering lower quality for relatively strong network effects and $c<0.053$.

The results discussed in this section can be summarized as follows. Depending on $c$ and $\delta$, the network effect impacts the amount of environmental externalities as well as consumer surplus positively if it is very weak. For slightly higher network effects, firms can strategically exploit the quality effect and the resulting increase in aggregate profits compensates the decrease of externalities and consumer surplus at this range. Finally, if the network effect is relatively strong, but not too strong (i.e. weak enough for the equilibrium discussed in Lemma 1 to exist), the loss of consumer surplus offsets increased profits and total welfare decreases in the network effect.

\section{Minimum Environmental Quality Standards}

In this section, we discuss the optimal choice of minimum environmental quality standards (MEQS), given the results presented in Section 4. We evaluate how this choice affects the amount of positive environmental externalities in equilibrium.

We assume that there is a regulator who can perfectly monitor if firms comply with the MEQS and that firms who violate the MEQS have to leave the market, i.e. have zero profit. Our discussion focuses on green product varieties. We do not explicitly discuss minimum quality standards that regulate the brown variety. Our model with three product varieties allows for the interpretation of the MEQS as an eco-label. ${ }^{17}$

\footnotetext{
${ }^{17}$ Particularly, the MEQS can be considered to be equivalent to the label in that both policies require the goods to meet certain minimum requirements. Meeting these requirements distinguishes green goods from the brown good in the absence of uncertainty.
} 
As has been argued earlier, we are mainly interested in the situation where the network effect is relatively weak, i.e. $\lambda \in\left[0, \frac{2}{25} \bar{q}\right]$. Given this, an MEQS, $\underline{q}$, can only make a difference if it changes the quality decision of at least one firm, which implies that we leave the sphere of the equilibrium as discussed in Lemma 1. Particularly, if $\lambda \in\left[0, \frac{2}{25} \bar{q}\right]$ the quality choice of the low-green firm is affected by the MEQS if the following condition holds.

\section{Condition 2.}

$$
\underline{q}>q_{l}^{\prime}\left(q_{h}, \lambda\right)
$$

Given that Condition 2 holds and $\lambda \in\left[0, \frac{2}{25} \bar{q}\right]$, equilibrium qualities are given by

\section{Lemma 2.}

$$
\left(q_{l}^{\star}, q_{h}^{\star}\right)=(\underline{q}, \bar{q})
$$

In this section, we refer to this subgame-perfect equilibrium of maximal quality differentiation as mSPNE. We proceed by proving Lemma 2.

Proof. First, we argue that $\left(q_{l}^{\star}, q_{h}^{\star}\right)=(\underline{q}, \bar{q})$ are indeed best responses to each other. Subsequently, we show that there always exists an mSPNE where the green market is not covered. Finally, we prove that Lemma 2 holds even if the green market is covered.

To see that an MEQS satisfying $\underline{q}>q_{l}^{\prime}\left(q_{h}, \lambda\right)$ forces the low-green firm to produce $q_{l}=\underline{q}$, note first that in equilibrium, $q_{l}^{\star}=q_{l}^{\prime}\left(q_{h}, \lambda\right)$ maximizes the profit of the low-green firm by definition (see Lemma 1). This implies that for $q_{l}<q_{l}^{\prime}\left(q_{h}, \lambda\right)$, the low-green firm prefers to produce strictly higher quality, while for $q_{l}>q_{l}^{\prime}\left(q_{h}, \lambda\right)$ the opposite holds. Hence, for an MEQS, $\underline{q}>q_{l}^{\prime}\left(q_{h}, \lambda\right)$, the low-green firm always wants to stick to the MEQS, which therefore defines the minimum quality produced in the market (actually she would prefer to produce lower quality, if she could without having to leave the market).

Moreover, in Lemma 1, we argued that the selected quality of the high-green firm equals $q_{h}^{\star}=\bar{q}$ for $\lambda<\frac{2}{25} \bar{q}$. When product differentiation decreases due to the MEQS, 
price competition increases. This implies that the high-green firm is even more eager to produce maximum quality.

Next, in the proof of Lemma 1, we have shown that the green market is not covered for $\lambda \in\left[0, \frac{2}{25} \bar{q}\right]$. This implies that market coverage must be increasing in $\underline{q}$ because $\frac{\partial d^{\star}}{\partial \underline{q}}=\frac{p_{l}^{\star}-\lambda}{\left(\lambda-q_{l}^{\star}\right)^{2}}>0$ if and only if $\hat{\theta}_{l}=\frac{p_{l}^{\star}-\lambda}{q_{l}^{\star}-\lambda}>0$, given that Condition 1 holds. However, if $\underline{q}=q_{l}^{\prime}\left(q_{h}, \lambda\right)+\epsilon$, where $\epsilon$ may be an arbitrarily small number, the green market will not be covered (i.e. $\hat{\theta}_{l}>0$ ) at least for some (small) $\lambda \in\left[0, \frac{2}{25} \bar{q}\right]$. Hence, if the MEQS exceeds the equilibrium low-green quality by an arbitrarily small amount, there exists an mSPNE such that $\hat{\theta}_{l}>0$. These results hold for any $c \in[0,1)$.

For larger values of the MEQS, i.e. $q>q_{l}^{\prime}\left(q_{h}, \lambda\right)+\epsilon$, the green market will be covered because $\frac{\partial d^{\star}}{\partial \underline{q}}>0$. Because the covered market equilibrium is an equilibrium of maximum product differentiation too (see Appendix B), the mSPNE exists irrespective of whether the green market is covered or not. ${ }^{18}$

Based on Lemma 2, we can evaluate the impact of the MEQS on environmental externalities. Particularly, employing the mSPNE, we show the following.

Proposition 4. $\frac{\partial E}{\partial \underline{q}}>0$, i.e. the amount of positive environmental externalities generated in a maximum differentiation equilibrium is increasing in the MEQS.

Proof. Consider the $E$ function (1) in the context of the mSPNE:

$$
E=d_{l}^{*} \underline{q}+d_{h}^{*} \bar{q}
$$

First, consider an non-covered green market. From the proof of Lemma 2, we know that $\frac{\partial d^{\star}}{\partial \underline{q}}=\frac{p_{l}^{\star}-\lambda}{\left(\lambda-q_{l}^{\star}\right)^{2}}>0$ if and only if $\hat{\theta}_{l}=\frac{p_{l}^{\star}-\lambda}{q_{l}^{\star}-\lambda}>0$, given that Condition 1 holds. In (16), this implies that $d_{l}^{*}+d_{h}^{*}$ is increasing in $\underline{q}$. Because $q<\bar{q}$, and $\bar{q}$ is exogenous, the proposition holds if we have that $\partial d_{h}^{*} / \partial \underline{q}>0$. This was already established by Equation (9) in Section 3.2.

Next, based on insights from Lemma 2, consider a covered green market. In the covered market mSPNE, demand levels, $d_{l}^{\star}=\frac{1+c}{3}$ and $d_{h}^{\star}=\frac{2-c}{3}$, are independent of the

\footnotetext{
${ }^{18}$ The mSPNE represents one of the additional equilibria mentioned in Footnote 11 for larger values of $\lambda$, where the low-green firm does not have a real-valued reaction function.
} 
quality choice of the low-green firm and therefore independent of the MEQS, $\underline{q}$. Because, in addition, $\bar{q}$ is exogenous, $E$ is necessarily increasing in the MEQS and therefore the proposition holds. This completes the proof.

Finally, we discuss the impact of regulation of the MEQS on total welfare. Previous studies, e.g. Ronnen (1991) and Cramps and Hollander (1995), indicate that in the absence of network effects, and independently of the presence of environmental externalities, an MEQS increases total welfare depending on the cost of producing quality. Given that $E$ increases in the stringency of the MEQS as discussed in Proposition 4, and given that the network effect directly increases consumer surplus, as can be seen from Equation (15) (holding quality levels constant), our last result is intuitively appealing.

Corollary 2. The welfare maximizing MEQS, $\underline{q}^{\star}$, is increasing in the network effect, for all $\lambda \in\left[0, \frac{2}{25} \bar{q}\right]$.

In other words, stronger network effects call regulators to impose a stricter MEQS. Rather than providing a formal proof of this corollary, we discuss the intuition of how an MEQS may affect total welfare in the presence of the network effect. ${ }^{19,20}$

In Section 4, we showed that consumer surplus is lower for higher values of $\lambda$ due to the increase in product differentiation and the quality effect. We also indicated that increased product differentiation results in relatively higher prices (see also Section 3.2). An MEQS reduces product differentiation (Lemma 2) and thereby intensifies price competition. $^{21}$ The aggregate benefit of lower prices is larger for higher values of $\lambda$, because the mass of green consumers increases more in $\underline{q}$ if there is a strong demand effect, as discussed in Proposition 1. An increase in the mass of green consumers does not only intensify the total network effect as represented by the third term on the right hand side

\footnotetext{
${ }^{19}$ Here we briefly outline a possible approach to formally prove this. Corollary 2 can be written as $\frac{\partial q^{\star}}{\partial \lambda}>0$ where $\underline{q}^{\star}(\lambda) \equiv \arg \max \partial W(\lambda, \underline{q}) / \partial \underline{q}$. In other words, at $\underline{q}^{\star}$, we have $\partial W(\lambda, \underline{q}) / \partial \underline{q}=0$. A formal proof requires us to show that for lower values of $\lambda, \partial W(\lambda, \underline{q}) / \partial \underline{q}=0$, while for larger values of $\lambda, \partial W(\lambda, \underline{q}) \partial \underline{q}>0$ for a given $\underline{q}$. Hence, we require that $\partial^{2} W(\lambda, \underline{q}) / \partial \underline{q} \partial \lambda>0$.

${ }^{20}$ For the sake of brevity, we refrain from showing simulations to support Corollary 2.

${ }^{21} \mathrm{~A}$ similar result has been reported earlier in Ronnen (1991) and Cramps and Hollander (1995). Moraga-González and Padrón-Fumero (2002) find that fiercer price competition due to a stricter MEQS increases the generation of negative externalities.
} 
of Equation (15), but also contributes to an increase in E. Eventually, these effects lead to a stronger increase in total consumer surplus due to higher $q$ when $\lambda$ is larger.

An increase in $\underline{q}$ goes along with a reduction of aggregate profits for the same reason that causes consumer surplus to increase: the restriction of the ability of firms to differentiate their products. Yet, the decrease in prices due to lower differentiability is lower for larger values of $\lambda$. For larger values of $\lambda$, firms benefit from the demand effect because of increased sales even if they have less scope for product differentiation.

\section{Conclusion and Discussion}

We analyzed a model of vertical product differentiation in a non-covered market for green products with two product varieties. We find that, depending on the strength of the network effect, firms produce lower average quality. Ultimately, this can lead to a decrease in the amount of positive environmental externalities generated in equilibrium, which may even adversely affect total welfare. This situation occurs when the increase in aggregate profits of the firms is lower than the loss of consumer surplus due to the social network effect.

Our results are driven by the fact that green firms have an incentive to further differentiate their products when there is a social network effect. Specifically, the lowgreen firm sets lower quality than in absence of the network effect, thereby a) escaping tight competition with the high-green firm, and b) reducing production costs. At the same time, given that the network effect increases the utility of purchasing a green product variety, producing lower quality does not lead to a substantial loss of market share.

The implications of these results for finding optimal minimum environmental quality standards are as follows. An MEQS constrains the ability of firms to differentiate, which leads to lower aggregate profits. However, an increase in consumer surplus compensates the loss of aggregate profits such that an MEQS increases total welfare. Hence, if there is a network effect, regulators should choose a stricter minimum environmental quality standard. However, we did not cover all possible aspects of regulation in this paper. The 
discussion of taxes and subsidies on markets for green products constitutes an interesting extensions.

The results of our model depend strongly on our stylized assumptions on the nature of the social network effect. We close the paper by discussing five alternative specifications; some of these represent interesting extensions of the model at hand. First, not every consumer may benefit from the social network effect of green consumption in the same way. Contrarily, people might be more or less prone to these effects as indicated by Frey and Meier (2004). In order to account for this, the model can be extended by assuming that $\lambda$ is a random variable. Second, the utility of green consumers may be adversely affected by the demand of brown consumers. Particularly, consumers may suffer when they perceive brown consumers to be free-riders on their green consumption. Third, the strength of the social network effect may depend on the chosen green product variety. Notably, if a consumer chooses the high-green product, she might have a stronger benefit from the network effect compared to the low-green product. If there are two distinct network effects, they might counter-balance each other. Fourth, $\lambda$ may be endogenous, as firms may engage in advertising, which may allow them to influence the strength of the social network effect. Fifth and finally, if green consumption triggers snob effects, the utility of some consumers from purchasing a green product may be decreasing in the number of consumers that buy the same product variety.

\section{Appendices}

\section{A Domination of one parabola branch}

Maximization of the profit function of the low-green firm, Equation (10), yields

$$
q_{l}\left(q_{h}, \lambda\right)=\frac{ \pm\left(q_{h}-\lambda\right) \sqrt{25 \lambda^{2}-52 \lambda q_{h}+4 q_{h}^{2}}+2\left(3 \lambda q_{h}+q_{h}^{2}-2 \lambda^{2}\right)}{7 q_{h}-3 \lambda},
$$

if and only if $\lambda \in\left[0, \frac{2}{25} q_{h}\right]$. This reaction correspondence represents a hyperbola (see Figure 2).

We show that the Nash equilibrium qualities on the upper branch of the hyperbola in Equation (17) strictly dominate the solutions on the lower branch. Let us use 
$\left(q_{1 l}\left(q_{h}, \lambda\right), q_{2 l}\left(q_{h}, \lambda\right)\right)$ to denote the two branches of Equation (17), and let us assign subscripts such that $q_{1 l}\left(q_{h}, \lambda\right)>q_{2 l}\left(q_{h}, \lambda\right)$. Substituting these into the profit function, $\pi_{l}$, we evaluate whether or not

$$
\Delta \equiv \pi_{l}\left(q_{1 l}\right)-\pi_{l}\left(q_{2 l}\right)>0
$$

It turns out that

$$
\Delta=\frac{96 \lambda w(c-1)^{2}\left(\lambda-2 q_{h}\right)^{2}\left(3 \lambda-7 q_{h}\right)^{3}\left(25 \lambda-2 q_{h}\right)}{\left(q_{h}-\lambda\right)\left(2 q_{h}-w+\lambda\right)\left(w+\lambda+2 q_{h}\right)\left(w+13 \lambda-26 q_{h}\right)^{2}\left(w-13 \lambda+26 q_{h}\right)^{2}}>0,
$$

where $w \equiv \sqrt{25 \lambda^{2}-52 q_{h} \lambda+4 q_{h}^{2}}$ for all $\lambda \in\left[0, \frac{2}{25} q_{h}\right]$. Hence, $q_{1 l}\left(q_{h}, \lambda\right)$ always yields higher profits than $q_{2 l}\left(q_{h}, \lambda\right)$, and hence the low-green firm's reaction function $q_{l}^{\prime}\left(q_{h}, \lambda\right)=$ $q_{1 l}\left(q_{h}, \lambda\right)$.

Next, we discuss the reaction function of the high-green firm if $\lambda \in\left(\frac{23 q_{l}}{25}, 1\right]$. From optimization of $\pi_{h}$ with respect to $q_{h}$, we obtain,

$$
q_{h}\left(q_{l}, \lambda\right)=\frac{1}{8}\left(7 \lambda+3 q_{l} \pm \sqrt{25 \lambda^{2}+2 q_{l} \lambda-23 q_{l}^{2}}\right)
$$

Let us denote the two branches of this Equation as $\left(q_{1 h}, q_{2 h}\right)$, where $q_{1 h}>q_{2 h}$. Then the high-green producing firm's reaction function is given by $q_{2 h}\left(q_{l}, \lambda\right)$ if $\Delta \equiv \pi_{h}\left(q_{2 h}\right)-$ $\pi_{h}\left(q_{1 h}\right)>0$. Solving, we find that indeed

$$
\Delta=\frac{3(c-1)^{2}\left(\lambda-q_{l}\right)\left(\lambda+q_{l}\right)^{2}\left(25 \lambda-23 q_{l}\right) \sqrt{25 \lambda^{2}+2 q_{l} \lambda-23 q_{l}^{2}}}{\left(\sqrt{25 \lambda^{2}+2 q_{l} \lambda-23 q_{l}^{2}}-u-q_{l}\right)^{2}\left(\sqrt{25 \lambda^{2}+2 q_{l} \lambda-23 q_{l}^{2}}+\lambda+q_{l}\right)^{2}}>0 .
$$

\section{B The covered market SPNE}

Here, we briefly review the outcome of the game without the non-covered green market assumption, i.e. $\hat{\theta}_{l}=0$. First, we calculate equilibrium prices.

$$
\begin{aligned}
& p_{l}=\frac{q_{h}(1+c)+q_{l}(2 c-1)}{3}, \\
& p_{h}=\frac{q_{l}(c-2)+q_{h}(2 c+1)}{3}
\end{aligned}
$$

and demands,

$$
d_{l}=\frac{1+c}{3},
$$




$$
d_{h}=\frac{2-c}{3}
$$

Note that $d_{l}$ is increasing in $c$, while the opposite holds for $d_{h}$. The more costly it is to produce a green variety, the lower the demand for this variety. This implies that, given the monopolistic structure of the market, demand shifts to the low-green variety. Moreover, the derivative of the profit function $\pi_{l}$ w.r.t. $q_{l}$ is a negative constant, $-\frac{1}{9}(1+$ $c)^{2}$. Contrarily, the derivative of the profit function, $\pi_{h}$ w.r.t. $q_{h}$ is a positive constant.

In a covered green market, given our assumption of the strength of the network effect being independent of consumed quality, every consumer of a green product variety benefits in the same way from social network effects. Thus, the covered market SPNE, as modeled here, is neither affected by externalities nor by the network effect, and thereby represents a simplified version of the setting analyzed by Brécard (2013).

\section{References}

James Andreoni. Impure altruism and donations to public goods: A theory of warm-glow giving. The Economic Journal, 100(401):, 464-477, 1990.

Sangeeta Bansal and Shubhashis Gangopadhyay. Tax/subsidy policies in the presence of environmentally aware consumers. Journal of Environmental Economics and Management, 45(2):, 333-355, 2003.

B Douglas Bernheim. A theory of conformity. Journal of Political Economy, 102(5): 841-877, 1994.

Dorothée Brécard. Environmental quality competition and taxation in the presence of green network effect among consumers. Environmental and Resource Economics, 54 (1):1-19, 2013.

Fredrik Carlsson, Jorge H García and Åsa Löfgren. Conformity and the demand for environmental goods. Environmental and Resource Economics, 47(3):407-421, 2010.

Chong Ju Choi and Hyun Song Shin. A comment on a model of vertical product differentiation. The Journal of Industrial Economics, 40(2):229-231, 1992. 
Klaus Conrad. Price competition and product differentiation when consumers care for the environment. Environmental and Resource Economics, 31(1):1-19, 2005.

Klaus Conrad. Price competition and product differentiation when goods have network effects. German Economic Review, 7(3):339-361, 2006.

Claude Crampes and Abraham Hollander. Duopoly and quality standards. European Economic Review, 39(1):71-82, 1995.

Helmuth Cremer and Jacques-François Thisse. On the taxation of polluting products in a differentiated industry. European Economic Review, 43(3):575-594, 1999.

Flash Eurobarometer. Europeans' attitudes towards the issue of sustainable consumption and production. Flash Eurobarometer 256, The Gallup Organisation, at the request of the Directorate-General for the Environment of the European Commission, 2009 .

Clas Eriksson. Can green consumerism replace environmental regulation? A differentiated-products example. Resource and Energy Economics, 26(3):281-293, 2004.

Bruno S Frey and Stephan Meier. Social comparisons and pro-social behavior: Testing "conditional cooperation" in a field experiment. American Economic Review, 94(5): $1717-1722,2004$.

James W Friedman and Isabel Grilo. A market with a social consumption externality. The Japanese Economic Review, 56 (3): 251-272, 2005.

Matthew J Kotchen. Green markets and private provision of public goods. Journal of Political Economy, 114(4):816-834, 2006.

Michael Kuhn. Minimum quality standards and market dominance in vertically differentiated duopoly. International Journal of Industrial Organization, 25(2):275-290, 2007.

Harvey Leibenstein. Bandwagon, snob, and Veblen effects in the theory of consumers' demand. The Quarterly Journal of Economics, 64(2):183-207, 1950. 
José Luis Moraga-González and Noemi Padrón-Fumero. Environmental policy in a green market. Environmental and Resource Economics, 22(3):419-447, 2002.

Martin A Nowak, and Karl Sigmund. Evolution of indirect reciprocity. Nature, 437 (7063):1291-1298, 2005.

Uri Ronnen. Minimum quality standards, fixed costs, and competition. The RAND Journal of Economics, 22(4):490-504, 1991.

Reinhard Selten. Reexamination of the perfectness concept for equilibrium points in extensive games. International Journal of Game Theory, 4(1):25-55, 1975.

Avner Shaked and John Sutton. Relaxing price competition through product differentiation. The Review of Economic Studies, 49(1):3-13, 1982. 